\title{
Kinder und Jugendliche schützen dank Tabakwerbeverbot
}

\author{
Barbara Weil \\ Leiterin Abteilung Gesundheitsförderung und Prävention
}

Die Ärzteschaft fordert ein umfassendes und striktes Werbe-, Promotions- und Sponsoringverbot für Tabakprodukte und E-Zigaretten. Sollen der durch den Tabakkonsum verursachte gesundheitliche Schaden, der Verlust an Lebensqualität und das Leid effizient eingedämmt werden, müssen künftig - insbesondere zum Schutz der Kinder und Jugendlichen - alle Lebensbereiche frei von Tabakwerbung sein.

\section{Die Ausgangslage}

In der Schweiz rauchen aktuell 25\% der Bevölkerung 9000 Menschen sterben jedes Jahr an den Folgen des Tabakkonsums. Die medizinischen und volkswirtschaftlichen Kosten belaufen sich jährlich auf 10 Milliarden Franken. Wenn Jugendliche bis zum 21. Lebensjahr nicht mit dem Rauchen angefangen haben, rauchen sie mit grosser Wahrscheinlichkeit ihr Leben lang nie. 57\% der Rauchenden fangen als Minderjährige mit dem Tabakkonsum an.

\section{Die Ärzteschaft fordert ein umfassendes und striktes Werbe-, Promotions- und Sponsoring- verbot für Tabakprodukte und E-Zigaretten.}

Im Gegensatz zur Schweiz haben bisher 180 von 192 Mitgliedstaaten der Weltgesundheitsorganisation WHO die internationale Rahmenkonvention über Tabakkontrolle (Framework Convention on Tobacco Control FCTC) ratifiziert. Vielen anderen Ländern gelingt es, die Zahl der Raucherinnen und Raucher dank umfassenden und strikten Werbereglementierungen signifikant zu verringern.

Für Tabakprodukte und E-Zigaretten mit oder ohne Nikotin ist deshalb ein umfassendes Werbe-, Promotions- und Sponsoringverbot einzuführen. Alle Lebensbereiche sollen frei von Tabakwerbung werden. Das bedingt unter anderem ein Verbot der Werbung und der Verkaufsförderung durch Rabatte.

\section{Die Argumente}

Die folgenden drei Punkte sind essentiell, um die Zahl der Raucherinnen und Raucher in der Schweiz und die gesundheitlichen Folgen des Rauchens nachhaltig zu reduzieren:

\section{Nur ein umfassendes Werbeverbot für Tabak- produkte ist wirksam}

Es ist unverantwortlich, dass Tabakwerbung zum Beispiel am Kiosk nach wie vor zugelassen ist, an Orten also, an denen Zigaretten via Bildschirme, Plakate, Verkaufsständer etc. angepriesen werden, oftmals genau auf Kinderaugenhöhe, zwischen Kaugummis und Bonbons. An Schweizer Open Airs können Jugendliche zwischen zwei Konzerten neue Zigaretten testen und sich mit Gadgets der Tabakindustrie eindecken lassen. Aktuell macht die Werbung am Verkaufsort rund die Hälfte der Vermarktungsmassnahmen der Tabakindustrie aus. Bei einem nur teilweisen Werbeverbot ist in Zukunft eine Verlagerung der Werbemittel an die Verkaufsorte zu erwarten.

\section{Hürden für Kinder und Jugendliche zum Erwerb von Tabakprodukten erhöhen}

Der Jugendschutz muss wirksam gestaltet werden: Das Verkaufsverbot für Minderjährige und die Testkäufe sind deshalb zu ergänzen mit einer Lizenzierung der Tabakverkaufsstellen. Ebenfalls ist der Verkauf von Tabakwaren über Automaten zu untersagen. Die einfache Erhältlichkeit nikotinhaltiger E-Zigaretten für Kinder und Jugendliche darf diese nicht zur Eingangspforte in die Nikotinsucht werden lassen. Deshalb ist es wichtig, dass E-Zigaretten mit Nikotin den gleichen Bestimmungen unterliegen wie die anderen Tabakprodukte.

\section{Attraktivität durch Einheitspackungen bei \\ Tabakwaren senken}

In Australien dürfen seit Dezember 2012 Tabakwaren nur in werbefreien Einheitspackungen verkauft werden. Erste Forschungsresultate bestätigen, dass Einheitspackungen die Attraktivität des Rauchens verkleinern und den Aufhörwunsch verstärken. Nach Australien hat Irland als zweites Land grünes Licht gegeben für werbefreie Verpackungen; Grossbritannien und weitere Staaten prüfen die Einführung. Der Schweizer Bundesrat schlägt vor, Kennzeichnung und 
Warnhinweise wie bisher in der Tabakverordnung zu regeln.

Die Verpackung einer Ware dient zugleich der Werbung. Die Tabakindustrie nützt dies, um ihre Produkte besonders für ausgewählte Zielgruppen attraktiver zu machen. Deshalb ist das Tabakproduktegesetz so auszugestalten, dass die Einführung von Einheitspackungen in der zukünftigen Tabakverordnung grundsätzlich möglich ist. Zumindest sollten die bisherigen Vorschriften an die neue EU-Richtlinie angeglichen werden.

Die kombinierten Warnhinweise mit Text und Bild sollen auf beiden Breitseiten je 80 Prozent belegen. Zudem sind die Warnhinweise in der oberen Hälfte der Verpackung anzubringen. Sinnvoll ist es ebenfalls, dass in der zukünftigen Tabakverordnung die Vorgaben der EU-Richtlinie zu Grösse und Form der Packungen übernommen werden. So können beispielsweise Werbeaktionen mit extra schmalen Packungen verhindert werden, die unterschwellig suggerieren, dass Zigaretten schlank machen.

Völlig ungenügend ist ein Sponsoringverbot von Tätigkeiten und Veranstaltungen, die international aus- gerichtet sind oder grenzüberschreitende Wirkung haben, wie dies im Gesetz vorgesehen ist. Jegliches Sponsoring soll untersagt werden.

\section{Unsere Forderungen}

Aus diesen Gründen fordern die FMH und die unterzeichnenden Ärzteorganisationen:

- Ein umfassendes und striktes Werbe-, Promotionsund Sponsoringverbot von Tabakprodukten: Keine Tabakwerbung, sei es in Printmedien, im Internet und in sozialen Medien oder auf Plakaten, in Kinos und an Verkaufsstellen.

- Keine direkte oder indirekte Verkaufsförderung von Tabakwaren z.B. durch Rabatte, Wettbewerbe oder Werbespiele.

- Kein Sponsoring öffentlicher sowie privater Anlässe durch Tabakfirmen.

- Klare Einschränkung der Attraktivität durch Einheitspackungen bei Tabakwaren (plain packaging).
Dieses Positionspapier erläutert die gemeinsame Haltung der FMH sowie folgender Ärzteorganisationen:

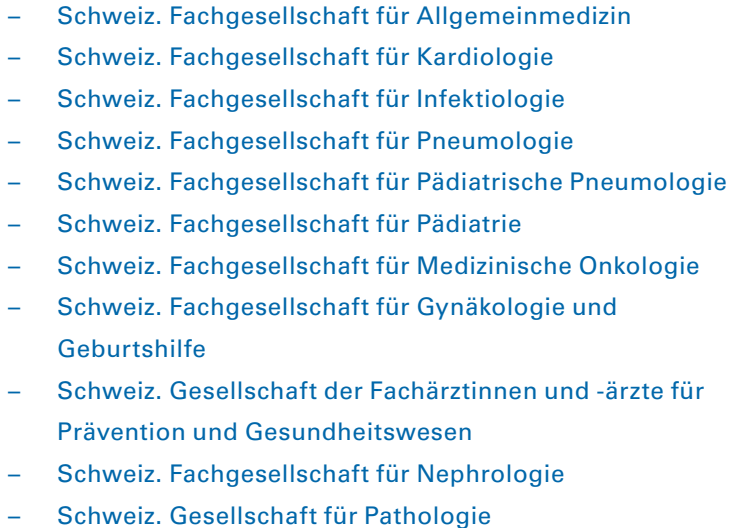

- Verband Schweizer Assistenz- und Oberärztinnen und -ärzte (VSAO)

- Kollegium für Hausarztmedizin (KHM)

- Haus- und Kinderärzte Schweiz (FME)

- Konferenz der Kantonalen Ärztegesellschaften (KKA)

- Verein der Leitenden Spitalärzte der Schweiz (VLSS)

- Swiss Medical Students' Association (SWIMSA)

- Walliser Ärzteverband

- Ärztegesellschaft des Kantons Zug

- Association des Médecins du canton de Genève

- Unterwaldner Ärztegesellschaft

- Société Vaudoise de Médecine 